Jean-Paul Renne*

\title{
A model of the euro-area yield curve with discrete policy rates
}

DOI 10.1515/snde-2016-0043

\begin{abstract}
This paper presents a no-arbitrage yield-curve model that explicitly incorporates the central-bank policy rate. This model is consistent with the existence of a lower bound for nominal interest rates, which makes it particularly relevant in the current context of extremely low interest rates. Changes in the policy rates depend on the monetary-policy phase, that can be either in an easing, status quo or tightening mode. The estimation of the model, based on daily euro-area yield data, reveals the strong influence of the monetary-policy phases on the shape of the yield curve. This relationship can, in turn, be exploited to estimate the probabilities of being in the different monetary-policy phases. The model is also used to compute term premiums, that are the parts of the yields reflecting the aversion of investors to interest rate risk. The results point to the existence of statistically significant premiums for many dates, even for short horizons.
\end{abstract}

Keywords: affine term-structure models; zero lower bound; regime switching models.

JEL Classification: E43; E44; E47; E52; G12.

\section{Introduction}

The standard view of the monetary policy transmission mechanisms suggests that central banks' actions are mainly transmitted to the economy through their effect on market interest rates. According to this standard view, a restrictive monetary policy pushes up both short-term and long-term interest rates, leading to less spending by interest-sensitive sectors of the economy, and vice versa. While there is a strong empirical support for the assertion that monetary policy is a major driver of the yield-curve fluctuations, a very few term-structure models explicitly incorporate the "policy rate" - the main central bank's instrument.

This paper proposes a novel and tractable no-arbitrage term-structure model where discrete-size changes in the monetary-policy rate are explicit and central. This framework is particularly adapted to depict the dynamics of the short-end of the yield curve, where the influence of monetary policy decisions is the most evident [see Cochrane and Piazzesi (2002)]. The estimation, carried out on euro-area daily data, sheds light on the influence of the ECB monetary policy on the term-structure of interest rates. Notably, consistently with the findings of Bikbov and Chernov (2008), my results show that the shape of the yield curve closely relates to the monetary-policy phase (easing, status quo or tightening). ${ }^{1}$ In turn, this relationship can be exploited to estimate the probabilities of being in the (unobserved) monetary-policy regimes.

The model is consistent with the fact that agents are averse to the interest rate risk. If this was not the case, the expectation hypothesis would hold. Under this hypothesis, long-term rates correspond to the average of expected future short-term rates [see Campbell and Shiller (1991) or Cochrane and Piazzesi (2005)]. While there is strong evidence against the expectation hypothesis for long-term yields, the evidence is weaker for

1 The definition of these phases is consistent with observed central banks' target-setting behavior and communication (see Smaghi 2009).

*Corresponding author: Jean-Paul Renne, University of Lausanne, Faculty of Business and Economics (HEC), CH1015 Lausanne Switzerland, e-mail: jean-paul.renne@m4x.org 
short-term ones [see Longstaff (2000)]. In my model, the possible departure from the expectation hypothesis results from the existence of a stochastic discount factor (s.d.f.). Thanks to this feature, term premiums can be computed. These premiums are defined as the differences between actual (observed) yields and the (counterfactual) yields that would be observed if agents were risk-neutral. ${ }^{2}$ Even for short maturities, I obtain term premiums that, for many dates, are statistically significant. In other words, my results provide evidence against the expectation hypothesis for short-term yields.

The model involves a lot of Markovian regimes - more than 100. Indeed, in the framework, a regime is characterized by (a) the level of the policy rate (about 40 in the present version of the model) 3 and (b) the monetary policy phase (easing, status quo or tightening). This distinguishes the present framework from earlier interest rate models involving regime switching. ${ }^{4}$ In spite of this unusual feature, the approach remains tractable both in terms of bond pricing and of estimation. This is illustrated by estimating the model on euro-area daily data covering the period from January 1999 to February 2012. The shortest-term rate I consider in the empirical exercise is the interbank overnight interest rate, which most central banks aim at stabilizing to a level close to a policy (or target) rate. Consistently with this choice, overnight index swap (OIS) rates are used as longer-term yields. An OIS is a fixed-for-floating interest rate swap with a floating rate leg tied to the index of daily interbank rates. The OIS curve is more and more seen by market participants as a proxy of the risk-free yield curve [see Joyce et al. (2011) and BIS (2013)]..$^{5}$ OIS have become popular hedging and positioning vehicles in euro financial markets and grew significantly in importance during the financial turmoil of the last few years.

The model is estimated by maximum likelihood techniques. The computation of the log-likelihood is based on an innovative joint use of the Kitagawa-Hamilton's filter and of the so-called inversion techniques originally introduced by Chen and Scott (1993).

In a companion paper [Renne (2016)], I use a simpler version of the present model to price interest rate options. However, in this previous paper, I do not explicitly model the stochastic discount factor. That is, in Renne (2016), the analysis is carried out under the risk-neutral world only, thereby ruling out the computation of term premiums. Other papers that closely relate to the present one are those by Piazzesi (2005) and Fontaine (2009). In both papers, the authors propose term-structure models that explicitly involve the target for the policy rate. They estimate their models on U.S. data covering, respectively the periods 1994-1998 (weekly) and 1994-2007 (daily). A common drawback of these frameworks is that they do not preclude negative policy rates. While this caveat may be tenable when the short-term interest rate is far enough from zero - the conditional probabilities of having negative interest in the subsequent periods being negligible-, it is more problematic in the current context of very low interest rates [see e.g. Christensen and Rudebusch (2013)]. More generally, many of the tractable yield-curve models are not consistent with the ZLB restriction [see Dai and Singleton (2003) or Piazzesi (2010)]. ${ }^{6}$ This limitation is addressed in the present framework.

2 Specifically, the latter yields are calculated by setting the estimates of the so-called prices of interest rate risk - that define the s.d.f. - to zero. These yields are those that would prevail under the expectation hypothesis.

3 The modeled policy rate is valued in a set of positive multiples of 25 basis points (or 0.0025). Indeed, over the estimation period (1999-2012), all ECB policy rates were multiples of 25 basis points. On 11 June 2014, though, the governing council of the ECB has set the rate of the main refinancing operations at $0.15 \%$ (see https://www.ecb.europa.eu/stats/monetary/rates/html/index. en.html). Such an event was not taken into consideration by markets before 2012. If the model was to be used for the most recent period, it could easily be adjusted to accommodate such values of the policy rates.

4 Seminal contributions include those of Hamilton (1988), Garcia and Perron (1996), Gray (1996), Bansal and Zhou (2002), Dai, Singleton, and Yang (2007), Ang, Bekaert, and Wei (2008) or Lemke and Archontakis (2008).

5 To that respect, one can note that German sovereign bonds, usually perceived as being the European "safest haven" both in terms of credit quality and liquidity, trade at levels that have remained close to the OIS yield curve over the last years. The fact that OIS rates are proxies of risk-free yields allows me not to consider the pricing of credit risk in the model.

6 Andreasen and Meldrum (2011) illustrate the ability of quadratic term structure models (QTSM) to preclude negative interest rates. These models are tractable but cannot accommodate long periods of unchanged interest rates; the same drawback characterises Cox, Ingersoll, and Ross (1985)'s model [see e.g. Kim and Singleton (2012)]. By contrast, the shadow-rate approach is consistent with prolonged ZLB periods. Nevertheless, this approach, originally proposed by Black (1995) and recently revived by e.g. Krippner (2013) or Christensen and Rudebusch (2015), does not entail explicit bond-pricing formula. Hence, working with shadow-rate models requires numerical approximations or simulations [see e.g. Priebsch (2013), Kim and Priebsch (2013) or Wu and Xia (2013)]. 
Indeed, in my model, the probability of a cut in the policy rate is zero when this rate is at its lower bound, thereby precluding negative policy rates.

The remainder of the paper proceeds as follows. Section 2 develops the model. Section 3 presents the data. Section 4 details the estimation strategy and results. Section 5 concludes.

\section{The model}

This section formulates a model of the daily dynamics of the overnight interbank interest rate. ${ }^{7}$ also specify a parametric stochastic discount factor and derive the resulting bond prices.

\subsection{The components of the overnight interest rate}

The policy rate prevailing at date $t$ is denoted by $\bar{r}_{t}$. A is the case in most currency areas, this policy rate is assumed to be a multiple of $0.25 \%$. I proceed under the assumption that the target rate is lower than a maximal rate denoted by $r_{\max }$ and equal to $0.25 \% \times N$, say. Therefore:

$$
\bar{r}_{t}=\Delta^{\prime} z_{r, t},
$$

where $z_{r, t}$ is a selection vector - that is one of the columns of $I_{N+1}$, the identity matrix of dimension $(N+1) \times(N+1)-$ and where the entries of the vector $\Delta$ are the continuously-compounded possible policy rates. ${ }^{8}$

The interbank overnight interest rate is denoted by $r_{t}$. Its deviations from the target rate are accounted for by two independent components, denoted by $\xi_{t}$ and $s_{t}$. Formally:

$$
r_{t}=\bar{r}_{t}+s_{t}+\xi_{t} \text {. }
$$

\subsubsection{The dynamics of the target rate $\bar{r}_{t}$}

Central bankers can decide to change the policy rate at their regular meetings. On these dates, the target can be raised (respectively cut) if the tightening regime (resp. the easing regime) prevails. By constrast, the policy rate remains necessarily unchanged under the status quo regime. The monetary regime is represented by a three-dimensional selection vector $z_{m, t}$ that is valued in the set of the three columns of the identity matrix $I_{3}$, corresponding, respectively to the tightening, the status quo and the easing regimes. Contrary to the econometrician, market participants observe the regime, this knowledge being based on a variety of detailed policy-relevant information that is not modeled here.

It is easily seen that the Kronecker product of the selection vectors $z_{r, t}$ and $z_{m, t}$, denoted by $z_{t}$, is also a selection vector that is valued in the set of the columns of $I_{3(N+1)}$. (Recall that $N+1$ is the number of possible values of the target rate, between $0 \%$ and $r_{\max }=N \times 0.25 \%$.) The dynamics of $z_{t}$ is described by a Markov chain. The matrix of transition probabilities of $z_{t}$ is denoted by $\Pi_{t}$. These matrices are time-inhomogenous, but in a deterministic way. Indeed, the matrices $\Pi_{t}$ can take two values, one of them being specific to those days at which a monetary-policy meeting is scheduled..$^{9}$ The number of entries of these $\Pi$ matrices is considerable: for $r_{\max }=10 \%$, there are 15.129 of them. Nevertheless, owing to the following assumptions, most of these entries are zero:

7 The extension to a lower frequency is straightforward.

8 Specifically, using the money-market day-count convention, the $i$ th entry of $\Delta$ is given by $\log (1+(i-1) 0.25 \% / 360)$.

9 Contrary to the policy rate $\left(z_{r, t}\right)$, that can change only following a monetary-policy meeting, the monetary-policy regime $\left(z_{m, t}\right)$ can switch at any date. 
1. Conditionally on being in an easing, a status quo or a tightening regime, the target moves are respectively valued in $\{-0.50 \%,-0.25 \%, 0\},\{0\}$ and $\{0,+0.25 \%,+0.50 \%\}$.

2. Easing or tightening phases are necessarily followed by status quo phases.

Even with these restrictions, many of $\Pi_{t}$ 's entries still require to be parameterised. Eight sets of probabilities have to be defined: two of them contain the probabilities of switching to the status quo regime (the probability of exiting the easing and the tightening regimes are respectively denoted by $p_{E S}$ and $p_{T S}$ ), two others are the probabilities of exiting the status quo regime $\left(p_{S E}\right.$ and $p_{S T}$ ), two of them contain the probabilities of 25-bp changes in the target rate (rise: $p_{r 25}$; cut: $p_{c 25}$ ) and the last two are the probabilities of 50-bp moves (rise: $p_{r 50}$; cut: $p_{c 50}$. These probabilities may vary with the policy rate. For instance, the probability of switching from the tightening to the status quo regime could be larger for higher policy rates, say. In order to keep the model parsimonious, the transition probabilities are based on logit parametric functions of the target rate $\bar{r}$. Formally, let me define the function $f$ by:

$$
f\left(\bar{r},\left[a_{1}, a_{2}\right]^{\prime}\right)=\left[1+\exp \left(a_{1}+a_{2} \bar{r}\right)\right]^{-1} .
$$

For $i \in\{T S, E S, S E\}$, the probabilities $p_{i}$ are characterized by 2-dimensional vectors of parameters $\alpha_{i}$ and are given by $f\left(\bar{r}, \alpha_{i}\right)$. Further, so as to have $p_{S T}+p_{S E}<1$, the probabilities $p_{S T}$ are defined by $\left(1-p_{S E}(\bar{r})\right) f\left(\bar{r}, \alpha_{S T}\right)$. Moreover, $\alpha_{i}$-type vectors are not defined for each of the four kinds of target moves $( \pm 0.25 \%, \pm 0.50 \%)$ but only for two: one for the rises in the policy rate $\left(\alpha_{r}\right)$ and one for the cuts $\left(\alpha_{c}\right)$. Two additional parameters, $k_{r}$ and $k_{c}$, are then introduced to share the rise and cut probabilities into those of 25-bp and 50-bp moves. Formally, the conditional probabilities of target-rate changes (i.e. $p_{c 25}, p_{r 25}, p_{c 50}$ and $p_{r 50}$ ) are defined through:

$$
\begin{gathered}
\left\{\begin{array}{lll}
p_{c 25}(\bar{r})=k_{c} f\left(\bar{r}, \alpha_{c}\right) & \text { and } p_{c 50}(\bar{r})=\left(1-k_{c}\right) f\left(\bar{r}, \alpha_{c}\right) & \text { if } \bar{r} \geq 0.50 \% \\
p_{c 25}(\bar{r})=f\left(\bar{r}, \alpha_{c}\right) & \text { and } p_{c 50}(\bar{r})=0 & \text { if } \bar{r}=0.25 \% \\
p_{c 25}(\bar{r})=0 & \text { and } p_{c 50}(\bar{r})=0 & \text { if } \bar{r}=0 .
\end{array}\right. \\
\left\{\begin{array}{lll}
p_{r 25}(\bar{r})=k_{c} f\left(\bar{r}, \alpha_{c}\right) & \text { and } p_{r 50}(\bar{r})=\left(1-k_{c}\right) f\left(\bar{r}, \alpha_{c}\right) & \text { if } \bar{r} \leq r_{\max }-0.50 \% \\
p_{r 25}(\bar{r})=f\left(\bar{r}, \alpha_{c}\right) & \text { and } p_{r 50}(\bar{r})=0 & \text { if } \bar{r}=r_{\text {max }}-0.25 \% \\
p_{r 25}(\bar{r})=0 & \text { and } p_{r 50}(\bar{r})=0 & \text { if } \bar{r}=r_{\text {max }} .
\end{array}\right.
\end{gathered}
$$

where $k_{c}$ and $k_{r}$ are valued in $[0,1] .^{10}$

Eventually, the 15.129 entries of matrix $\bar{\Pi}$ are defined by 14 parameters only.

\subsubsection{The volatile component $\xi_{t}$}

Factor $\xi_{t}$ is aimed at capturing the volatile and short-lived (noise) fluctuations of the spread between the interbank overnight rate and the policy rate. I assume that the $\xi_{t}$ s are independent exogenous random variables. The distribution of these variables is denoted by $\mathscr{L}$; this distribution is designed in such a way as to flexibly accommodate non-zero skewness and fat tails. Specifically, a random variable drawn from distribution $\mathscr{L}\left(w, p, \alpha_{P}, \beta_{P}, \alpha_{N}, \beta_{N}\right)$ is equal to $w+\mathbb{I}_{\{u=0\}} v_{P}-\mathbb{I}_{\{u=1\}} v_{N}$, where $u$ is Bernoulli distributed with success probability $p$ and where $v_{P}$ and $v_{N}$ follow $\beta$ distributions with parameters $\left(\alpha_{P}, \beta_{P}\right)$ and $\left(\alpha_{N}, \beta_{N}\right)$, respectively.

10 Before November 2001, possible changes in the policy rate were discussed in each of the bi-weekly meetings of the ECB Governing Council. Since then, they are considered during the first of these two bi-weekly meetings only. Accordingly, for the first part of the sample (up to November 2001), the target-moves probabilities are divided by two so as to result in (approximately) the same probabilities of target moves over a month. 


\subsubsection{The dynamics of $s_{t}$}

The variable $s_{t}$ is aimed at contributing to persistent fluctuations of yields that cannot be accounted for by the regime variables $z_{r, t}$ and $z_{m, t^{*}}$ Combined with $\xi_{t}$, the regime variables are expected to account for most of EONIA's fluctuations. Therefore, the variable $s_{t}$ is expected to have a far lower impact on the overnight rate than on longer-term yields. To obtain such a feature (without resorting to an explosive dynamics for $s_{t}$ ), $s_{t}$ is decomposed into two components denoted by $s_{1, t}$ and $s_{2, t}$, that is, $s_{t}=s_{1, t}+s_{2, t^{1}}{ }^{11}$ The dynamics of $\left[s_{1, t} s_{2, t}\right]^{\prime}$ is defined through:

$$
\begin{gathered}
{\left[\begin{array}{l}
s_{1, t} \\
s_{2, t}
\end{array}\right]=\Phi\left[\begin{array}{l}
s_{1, t-1} \\
s_{2, t-1}
\end{array}\right]+\Sigma \varepsilon_{t}, \quad \varepsilon_{t} \sim \text { i.i.d. } \mathcal{N}(0, I)} \\
\text { where } \Phi=\left[\begin{array}{cc}
\rho_{1} & \beta \\
0 & \rho_{2}
\end{array}\right] \text { and } \Sigma=\left[\begin{array}{cc}
0 & 0 \\
0 & \sigma_{s}
\end{array}\right] .
\end{gathered}
$$

The smaller $\beta$, the less variable $s_{1, t}$ is. In the limit, if $\beta$ is equal to zero and if $s_{1, t}$ was zero at some point in the past, then $s_{t}=s_{2, t}$. I assume this is the case under the physical measure, but not under the risk-neutral one. ${ }^{12}$ Under the latter measure, if the $\rho_{i}^{* \text { 's }}$ - the risk-neutral counterpart of the $\rho_{i}^{\text {'s }}$ - are close to one, a shock on $s_{2, t}$ can have a very persistent impact on $s_{t}$. In addition, if $\beta^{*}$ is large enough, these effects are multiplied by feeding through $s_{1, t}$. Therefore, $s_{t}$ 's innovations may have a far more long-lasting impact under the risk-neutral measure than under the physical measure. This implies that $s_{t}$ may account for a far larger variance of longterm yields than of short-term yields. ${ }^{13}$

\subsection{Pricing}

\subsubsection{The stochastic discount factor (s.d.f.)}

I assume that the stochastic discount factor (s.d.f.) between dates $t-1$ and $t$ is given by:

$$
M_{t-1, t}=\exp \left(-r_{t-1}-\frac{1}{2} v_{t-1}^{\prime} v_{t-1}+v_{t-1}^{\prime} \varepsilon_{t}+\left(z_{t-1}^{\prime} \delta_{t}\right) z_{t}\right) \text {. }
$$

The vectors $\delta_{t}$ and $v_{t}$ are the prices of risk associated to the regime shifts and to the Gaussian shocks $\varepsilon_{t}$, respectively. Assuming that vector $v_{t}$ satisfies $\Sigma v_{t}=\left(\Phi^{*}-\Phi\right)\left[s_{1, t} s_{2,1}\right]^{\prime}$ and defining matrix $\Pi_{t}^{*}$ through $\Pi_{t, i j}^{*}=\Pi_{t, i j} \exp \left(\delta_{i, t}\right)$, it can be shown that the dynamics of $z_{t}$ and $s_{t}$ under the risk-neutral measure are of the same kinds as their physical counterparts except that matrices $\Pi_{t}$ and $\Phi$ are respectively replaced by $\Pi_{t}^{*}$ and $\Phi^{*} .^{14}$ (The riskneutral - or pricing - measure $\mathbb{Q}$ is defined through the Radon-Nikodym derivatives of $\mathbb{Q}$ with respect to the physical measure $\left.\mathbb{P}: d \mathbb{Q} / d \mathbb{P}=\exp \left(r_{t-1}\right) M_{t-1, t}\right)$

It is worth stressing that these specifications offer the possibility to price monetary-policy-related risks, this feature resulting from the presence of monetary-policy phases $\left(z_{m, t}\right)$ and target rates $\left(z_{r, t}\right)$ in the definition of the stochastic discount factor.

11 An alternative would be to assume that $s_{t}$ follows an AR(1) process. However, to capture the previous feature, the auto-regressive parameter should be larger than one. With a Gaussian $s_{t}$, this would imply infinitely large (positive or negative) long-term rates. By contrast, the process that is proposed here ensures that the variances of long-term yields do not explode with maturities.

12 The relationship between the physical and the risk-neutral dynamics is discussed below, in Subsection 2.2.1.

13 The choice of this dynamics builds on Dubecq and Gouriéroux (2011)

14 See Monfort and Renne (2013). In particular, the $p_{E S}^{*}, p_{S E}^{*}, p_{S T}^{*}, p_{T S}^{*}, p_{r 25}^{*}, p_{c 25}^{*}, p_{r 50}^{*}$ and $p_{c 50}^{*}$, that define the $\Pi_{t}^{*}$ 's matrices, are based on functions $f(\bar{r}, \cdot)$. Still using the superscript “"” to denote risk-neutral parameters, these probabilities depend on some vectors $\alpha_{i}^{*}$ (see end of Subsection 2.1.1). 


\subsubsection{Bond prices}

It is well-known that the existence of a positive stochastic discount factor is equivalent to the absence of arbitrage opportunities [Hansen and Richard (1987)] and that the price at $t$ of a zero-coupon bond with residual maturity $h$, denoted by $P(t, h)$, is given by:

$$
P(t, h)=E_{t}\left(M_{t, t+1} \times \ldots \times M_{t+h-1, t+h}\right)=E_{t}^{\mathbb{Q}}\left(\exp \left[-r_{t}-\ldots-r_{t+h-1}\right]\right),
$$

where $E_{t}^{\mathbb{Q}}$ denotes the expectation under the risk-neutral measure, conditional on the information available at date $t$. Substituting Equation (2) into Equation (8) leads to:

$$
P(t, h)=E_{t}^{\mathbb{Q}}\left(\exp \left[-\sum_{i=0}^{h-1}\left(\bar{r}_{t+i}+s_{t+i}+\xi_{t+i}\right)\right]\right) .
$$

Under the assumption that $\bar{r}_{t}, s_{t}$ and $\xi_{t}$ are independent processes, it comes:

$$
\begin{aligned}
P(t, h) & =\left(E_{t}^{\mathbb{Q}} e^{-\sum_{i=0}^{h-1} \bar{T}_{t+i}}\right)\left(E_{t}^{\mathbb{Q}} e^{-\sum_{i=0}^{h-1} \xi_{t+i}}\right)\left(E_{t}^{\mathbb{Q}} e^{-\sum_{i=0}^{h-1} s_{t+i}}\right) \\
& =P_{z}(t, h) \times P_{\xi}(t, h) \times P_{s}(t, h) \quad \text { (say). }
\end{aligned}
$$

The computations of $P_{z}(t, h), P_{\xi}(t, h)$ and $P_{s}(t, h)$ are detailed in Appendix B. Explicit formulas are available to compute these three terms, each of them turning out to be exponential affine in $\left(z_{t}^{\prime}, s_{t}\right)^{\prime}$. Accordingly, the yields associated with zero-coupon bonds of maturity $h$, denoted by $y(t, h)$, are of the form: ${ }^{15}$

$$
y(t, h)=G(t, h) z_{t}+A_{h}+B_{h} s_{t} .
$$

\section{Data and stylised facts}

The sample period is January 15, 1999 to February 17, 2012 (3416 dates). The shortest-maturity rate I will consider is the EONIA (Euro OverNight Index Average), that is the daily euro-area interbank rate. Longer-term rates are overnight indexed swap rates (OIS). An OIS is an interest rate swap whose floating leg is tied to an overnight rate - the EONIA in the euro-area case - compounded over a specified term. Six maturities are included in the estimation dataset (in addition to the overnight one): 1 month, 3 months, 6 months, 12 months, 2 years and 4 years. ${ }^{16}$ The policy rate and the EONIA series come from the ECB; longer-term yields are taken from Bloomberg. ${ }^{17}$

15 Using the notations of Appendix B, I have $A_{h}=A_{h}^{(\xi)}+A_{h}^{(s)}$. Note that the values of $A_{h}$ are not the same before and after 8 October 2008 because of the change in the distribution of $\xi_{t}$ on that break date (see Section 3). Therefore, the pricing formulas of $P_{\xi}(t, h)$ actually have to be used twice, with $\mathscr{L}_{\text {norm }}$ first and then with $\mathscr{L}_{\text {excess }}$ (yielding to two different sets of constants $A_{h} \mathrm{~s}$ ). For notational convenience, this is not expressed in Equation (10). Note eventually that the time-dependence of $G$ is deterministic; this time dependence relates to the (publicly-known) dates of the monetary-policy meetings.

16 OIS yields are not available for longer-than-one-year maturities before 2005. Before that date, I use EURIBOR swaps data in place of the 2-year and 4-year OIS yields. Before 2007, the short-term EONIA swaps and maturity-matching EURIBORs had extremely close variations before 2007. For each maturity (2-year and 4-year), I subtract the 2005-2006 IBOR-OIS average spread from the EURIBOR swap series used in the estimation before 2005, which is about 10 basis points (the standard deviation is smaller than 3 basis points).

17 All yields are expressed in continuously-compounded terms. Money-market rate are based on the ACT/360 day-count basis, the corresponding continuously compounded rates are therefore computed as $\ln (1+d \times r / 360) \times 365 / d$, where $r$ is the considered rate and where $d$ is the residual maturity of the instrument. Since the pricing formula presented in Subsection 2.2 are consistent with zero-coupon yields, that type of yields is computed using classic bootstrapping methods (for maturities larger than one year, i.e. 2 and 4 years). 
Panel A of Figure 1 compares the fluctuations of the policy rate with these of the EONIA. Changes in the policy rate are decided during the first of the bimonthly meetings of the ECB's Governing Council. On a daily scale, this implies a step-like behavior for the target rate. This plot also displays the two additional policy rates of the Eurosystem framework. These two rates constitute the bounds of the so-called corridor that brackets the main policy rate..$^{18}$ The lower bound of the corridor is the deposit-facility rate, that is the rate at which banks can deposit cash overnight with the Eurosystem. The upper bound is the lending-facility rate, at which counterparties can borrow funds overnight from the Eurosystem. No bank would agree (a) to lend to another bank at a yield lower than the one it can secure by using the ECB deposit facility - which is a creditrisk-free investment - or (b) to borrow at a rate higher than the one it can get by using the lending facility. This ensures that the EONIA lies within the bounds of the corridor.

Panel B of Figure 1 presents the EONIA spread, that is the yield differential between the overnight interbank rate and the main policy rate, which is the middle of the corridor. In the model, the EONIA spread is $r_{t}-\bar{r}_{t}=s_{t}+\xi_{t}$. This plot highlights the break in the relationships between these two rates that occurred in 2008. On
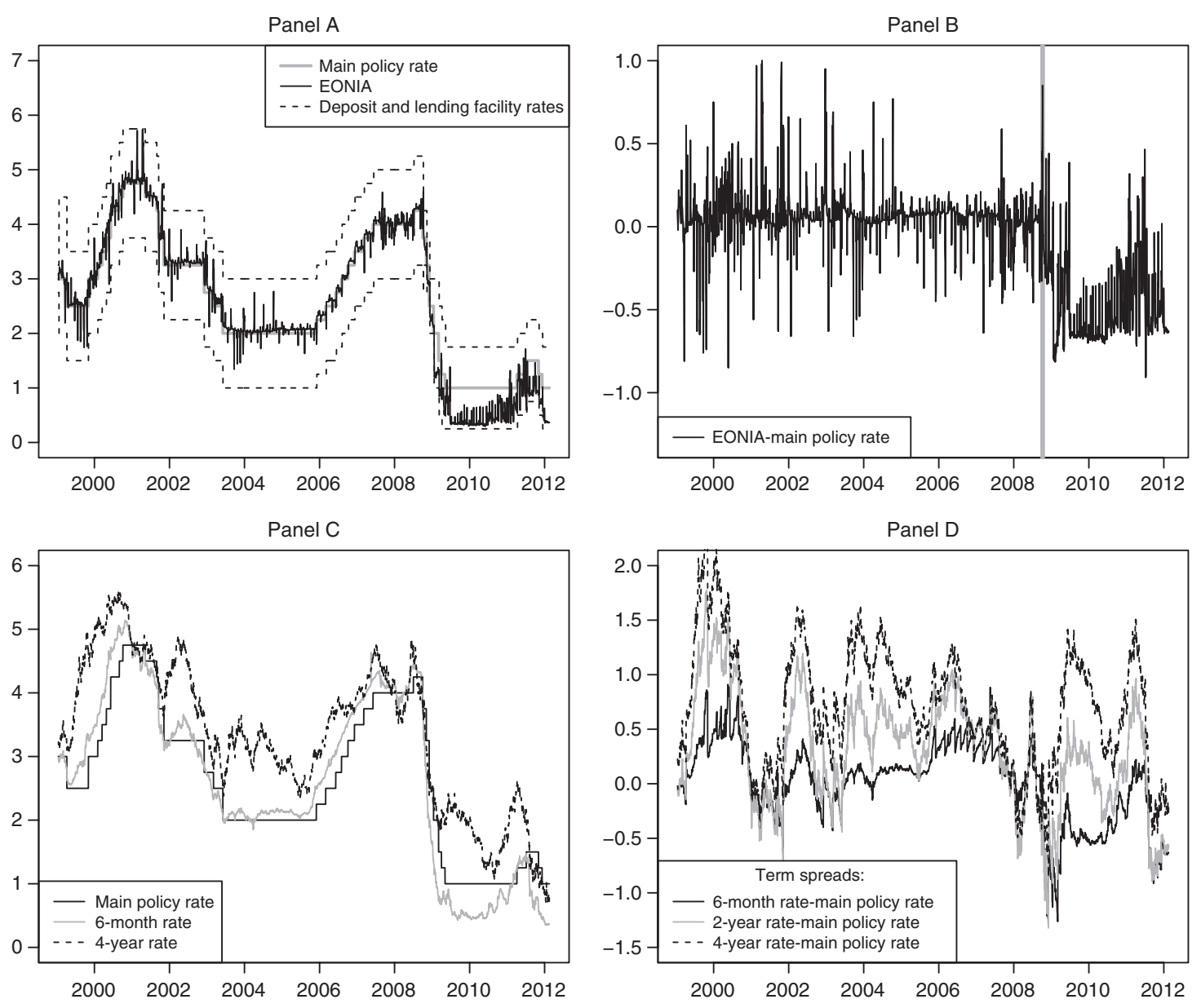

Figure 1: Target rate, EONIA and OIS.

Panel A shows the target rate together with the overnight interbank interest rate (EONIA). The dashed lines are the bounds of the corridor (see Subsection 3). Panel B displays the EONIA spread, i.e. the spread between the EONIA and the target. The vertical bar in Panel B indicates the date on which the Fixed-Rate Full Allotment procedures was announced by the ECB (8 October 2008). Panel C presents the target rate and two OIS yields. Spreads between OIS and the main policy rate are reported in Panel D.

18 See Kahn (2010) for a comprehensive description and an international comparison of "corridor” systems. 
8 October 2008, in a context of worldwide financial stress, the Eurosystem adopted a fixed-rate full allotment (FRFA) tender procedure. ${ }^{19}$ Since then, the ECB accommodates any demand for liquidity its bank counterparties might express at the policy rate - against eligible collateral - in unlimited amounts. This measure generated a steady excess of liquidity balances in the overnight market, putting downward pressure on the overnight interest rate. As a result, the latter rate drifted toward the lower limit of the monetary policy corridor [see Soares and Rodrigues (2013), Beirne (2012) and Fahr et al. (2010)]. In addition, it can be seen on Panel B that the distribution of the volatile component of the overnight rate became strongly positively skewed after the drop in the EONIA spread. Accordingly, the date of the FRFA announcement (8 October 2008) is treated as a break date. At that date, I allow for a change in the distribution of the volatile component $\xi_{t}$ of the EONIA rate. ${ }^{20}$ Specifically:

$$
\xi_{t} \sim\left\{\begin{array}{cl}
\mathscr{L}\left(w_{\text {norm }}, p_{\text {norm }}, \alpha_{P, \text { norm }}, \beta_{P, \text { norm }}, \alpha_{N, \text { norm }}, \beta_{N, \text { norm }}\right) & \text { for } t<8 \text { October 2008, } \\
\mathscr{L}\left(w_{\text {excess }}, p_{\text {excess }}, \alpha_{P, \text { excess }}, \beta_{P, \text { excess }}, \alpha_{N, \text { excess }}, \beta_{N, \text { excess }}\right) & \text { for } t \geq 8 \text { October } 2008 .
\end{array}\right.
$$

In particular, since the $\mathscr{L}$ distribution is not centred, the mean value of $\xi_{t}$ can differ in the two periods. (Looking at Panel B, it is expected to be close to zero before the break date and negative after.)

\section{Estimation}

\subsection{Estimation strategy}

The model is estimated by means of maximum likelihood techniques. In the spirit of Kalman filtering, the computation of the likelihood function is based on an algorithm that simultaneously derives estimates of the latent factors $\left(z_{t}\right.$ and $\left.s_{t}\right) \cdot{ }^{21}$

The model is a state-space model. Such a model entails two ingredients: (a) transition equations, which specifies the dynamics of the state vectors, and (b) the measurement equations, that describe the relationship between the state vectors and the observable variables. In the present case: (a) the dynamics of the state vectors $s_{t}$ and $z_{t}$ are respectively defined by Equation (6) and by the matrices of transition probabilities $\Pi_{t}$ and (b) the measurement equations relate observed yields, gathered in $R_{t}$ - a vector of $M$ observed yields of maturities $h_{1}, \ldots, h_{M}-$ to $s_{t}$ and $z_{t}$. Using Equation (11) and introducing some vectors of i.i.d. normal measurement errors denoted by $\eta$, the measurement equations, in vector form, are:

$$
R_{t}=\Lambda_{z} z_{t}+\Lambda_{s} s_{t}+\sigma_{R} \eta_{t}, \quad \eta_{t} \sim \mathcal{N}(0, I d) .
$$

At that stage, note that $z_{t}$ is only partially unobserved. Indeed, $z_{t}$ is the Kronecker product of $z_{r, t}$ and $z_{m, t}$; and $z_{r, t}$ is observed. Therefore, the true latent variables are $s_{t}$ and $z_{m, t^{\circ}}$ Equation (13) is accordingly rewritten as:

$$
R_{t}=\Lambda_{m, t} z_{m, t}+\Lambda_{s} s_{t}+\sigma_{R} \eta_{t},
$$

where $\Lambda_{m, t}$ is a $1 \times 3$ (observable) vector that is such that $\Lambda_{m, t} z_{m, t}=\Lambda_{z}\left(z_{r, t} \otimes z_{m, t}\right)^{22}$

19 See https://www.ecb.europa.eu/press/pr/date/2008/html/pr081008_2.en.html. The FRFA announcement date is indicated by a vertical bar on Panel B in Figure 1.

20 Alternatively, a modeling possibility is to introduce an additional regime-switching variable $\left(z_{\text {exc, }}\right)$ indicating whether the excess liquidity regime prevails, or not, at date $t$. The latter approach has been employed in a previous version of the paper. For the sake of simplicity, the breakpoint approach is used in the present one. The qualitative results are fairly robust to this simplification.

21 Since I assume that the EONIA rate is modeled without error, $\xi_{t}$ is not considered as a third unobserved variable. In other words, conditionally on the knowledge of $z_{t}$, s and $r_{t}$, variable $\xi_{t}$ is deterministic.

22 The $i$ th entry of $\Lambda_{m, t}$ is given by $\Lambda_{z}\left(z_{r, t} \otimes e_{i}\right.$, where $e_{i}$ denotes the $i$ th column of $I_{3}$, the identity matrix of dimension $3 \times 3$. 
This latency is handled by using an estimation strategy built on Monfort and Renne (2013). The approach consists in applying inversion techniques à la Chen and Scott (1993) together with the Kitagawa-Hamilton filter to address the hidden nature of the switching regimes. The idea of the inversion technique is the following: assuming that a linear combination of the yields is observed without error, one obtains the latent variable $s_{t}$ as a function of $R_{t}$ and $z_{t}$. In that case, one can compute the likelihood function; the calculation involves the specified dynamics of the latent factor as well as the distribution of the remaining pricing errors. Usually, one uses trivial perfectly-priced combinations of yields: specifically, if there are $m$ latent factors with continuous support in the model, one assumes that $m$ yields are priced without error. However, as noted for instance by Piazzesi (2010), the choice of these $m$ yields maturity, out of the $M$ available ones, is arbitrary. Therefore, I resort here to an original alternative approach and choose $s_{t}$ in order to minimize the average squared pricing errors across the different maturities. ${ }^{23}$ In that case, conditionally on $\left(R_{t}, z_{t}\right)$, the latent factor $s_{t}$ is simply obtained by using the OLS formula:

$$
s_{t}=\left(\Lambda_{s}^{\prime} \Lambda_{s}\right)^{-1} \Lambda_{s}^{\prime}\left(R_{t}-\Lambda_{z} z_{t}\right) .
$$

Equation (15) shows that $s_{t}$ is then given by a weighted average of the elements of $R_{t}-\Lambda_{z} z_{t}$, which are the parts of the yields that cannot be accounted for by $z_{t}$. Contrary to the classic inversion technique, in which there is a one-to-one relationship between one of the yields and $s_{t}$, the averaging underlying the present approach tends to make the results less sensitive to potential noises present in the yield observations (i.e. in $R_{t}$ ). Further details of the exact computation of the likelihood are provided in Appendix C.

\subsection{The estimated model}

Table 1 reports the maximum-likelihood parameter estimates..$^{24}$ The mean absolute pricing error is equal to 7 basis points, which is comparable, though slightly higher, to Piazzesi (2005)'s fit of the U.S. yield curve. ${ }^{25}$ Figure 2 shows the fit of the 6-month and the 4-year yields. These plots also show the part of those yields that is explained by the regime variable $z_{t}$. It appears that most of the yields' fluctuations can be accounted for by $z_{t}$ : more than $95 \%$ of the sample variances of yields with maturities lower than 2 years are captured by the term $G(t, h) z_{t}$ appearing in Equation (11). ${ }^{26}$

Figure 3 presents the estimated probabilities of being in the different monetary-policy regimes $\left(z_{m, t}\right){ }^{27}$ The policy rate is also plotted on this figure. Naturally, the chart shows that phases of cuts (respectively rises) in the policy rate take place when the easing (respectively tightening) regime prevails. Interestingly, it also appears that there are changes in the monetary policy regime that accompanied by movements of the policy rate. Such changes could be triggered by (non-modeled) ECB officials' speeches or by the release of macroeconomic news or figures. In the context of the model estimation, the detection of these changes is based on the information embedded in the shape of the yield curve. To illustrate, Figure 4 shows the influence

23 I am grateful to Simon Dubecq for providing me with this procedure. To my knowledge, though particularly efficient compared to classic inversion techniques, it has not been used in the existing literature.

24 In order to avoid that the factor $s_{t}$, thanks to its flexible Gaussian dynamics, explains too large a share of the yield fluctuations, I limit the size of its marginal variance in the estimation. Specifically, I impose that the marginal standard deviation of the $s_{t}$-related component of the 1-year yield is lower than 5 basis points. Eventually, 46 parameters remain to be estimated.

25 Piazzesi (2005)'s approach constitutes a good basis for comparison because the focus of her paper is put on the same spectrum of maturities as the present one. Note however that the sample period used by Piazzesi (2005) is shorter (4 years against 13 years here) and the frequency is lower (weekly vs. daily here). Another basis for the fit assessment is offered by principal component analysis: the mean absolute pricing error resulting from fitting the yields with their first two principal components is of 5 basis points ( 2 basis points with the first three principal components).

26 Eighty five percent of the variance of the 4-year yield is accounted for by $G(t, h) z_{t}$.

27 These probabilities are the smoothed probabilities computed with Kim (1994)'s algorithm. 
Table 1: Parameter estimates.

\begin{tabular}{|c|c|c|c|c|c|c|c|}
\hline & $a_{1}^{*}$ & $a_{2}^{*}$ & $\boldsymbol{k}^{*}$ & & $a_{1}$ & $a_{2}$ & $k$ \\
\hline \multirow[t]{2}{*}{ Rise in the policy rate } & -0.058 & 0.112 & 0.506 & & 1.035 & -0.157 & 0.889 \\
\hline & $(0.700)$ & $(0.024)$ & $(0.520)$ & & $(0.452)$ & $(0.026)$ & $(0.089)$ \\
\hline \multirow[t]{2}{*}{ Cut in the policy rate } & 0.254 & 0.102 & 0.147 & & 0.801 & -0.144 & 0.411 \\
\hline & $(0.320)$ & $(0.009)$ & $(0.329)$ & & $(0.546)$ & $(0.029)$ & $(0.142)$ \\
\hline \multirow[t]{2}{*}{ ES } & 3.260 & 0.518 & & & 5.173 & -0.415 & \\
\hline & $(0.056)$ & $(0.021)$ & & & $(0.696)$ & $(0.225)$ & \\
\hline \multirow[t]{2}{*}{ SA } & 8.980 & -0.879 & & & 6.039 & -0.473 & \\
\hline & $(1.000)$ & $(0.035)$ & & & (1.051) & $(0.286)$ & \\
\hline \multirow[t]{2}{*}{ TS } & 4.414 & 0.293 & & & 3.889 & 0.266 & \\
\hline & $(0.056)$ & $(0.015)$ & & & $(0.785)$ & $(0.278)$ & \\
\hline \multirow[t]{2}{*}{ ST } & 5.836 & -0.029 & & & 3.617 & 0.538 & \\
\hline & $(0.076)$ & $(0.051)$ & & & $(0.906)$ & $(0.091)$ & \\
\hline $\boldsymbol{\beta}_{P, \text { norm }}$ & $\boldsymbol{\beta}_{N, \text { norm }}$ & $\alpha_{P, \text { norm }}$ & $\alpha_{N, \text { norm }}$ & $w_{\text {norm }}$ & $\boldsymbol{p}_{\text {norm }}$ & & \\
\hline 0.760 & 0.178 & 8.192 & 1.643 & 0.040 & 0.571 & & \\
\hline$(0.033)$ & $(0.005)$ & $(0.129)$ & $(0.136)$ & $(0.001)$ & $(0.011)$ & & \\
\hline $\boldsymbol{\beta}_{P, \text { excess }}$ & $\boldsymbol{\beta}_{N, \text { excess }}$ & $\alpha_{P, \text { excess }}$ & $\alpha_{N, \text { excess }}$ & $w_{\text {excess }}$ & $p_{\text {excess }}$ & & \\
\hline 0.858 & 8.875 & 1.474 & 0.450 & -0.650 & 0.814 & & \\
\hline$(0.123)$ & (1.014) & $(0.038)$ & $(0.0375)$ & $(0.001)$ & $(0.010)$ & & \\
\hline$\rho_{1}^{*}$ & $\rho_{2}^{*}$ & $\beta^{*}$ & & $\rho_{2}$ & & $\sigma_{s}\left(\times 10^{6}\right)$ & $\sigma_{R}$ \\
\hline 0.999 & 0.998 & 105.511 & & 0.941 & & 0.930 & 0.091 \\
\hline$(0.001)$ & $(0.001)$ & $(0.534)$ & & $(0.001)$ & & $(0.007)$ & $(0.001)$ \\
\hline
\end{tabular}

The table reports the estimates of the parameters defining the dynamics of the factor under historical and risk-neutral measures. The estimation data are daily and span the period from January 1999 to February 2012. Standard errors are reported in parentheses. The parameters $a_{i}$ relate the probabilities of changes in the policy rate or in the monetary-policy regime to the level of the policy rate (see Subsection 2.1.1 and notably Equation 3). The parameters that define the risk-neutral dynamics are indicated by ${ }^{*} . \sigma_{R}$ is the standard deviation of the pricing errors (see Equation 13), expressed in percentage points. Parameters $w_{i}, p_{i}, \alpha_{p, i}, \beta_{p, i}, \alpha_{N, i}, \beta_{N, i}$ define the distribution of $\xi_{t}$, the volatile component of $r_{t}$ [see Equation (12)]. Parameters $\rho_{1}^{*}, \rho_{2}^{*}, \beta^{*}, \rho_{2}, \sigma_{s}$ characterize the dynamics of $s_{t}$ (see Subsection 2.1.3).
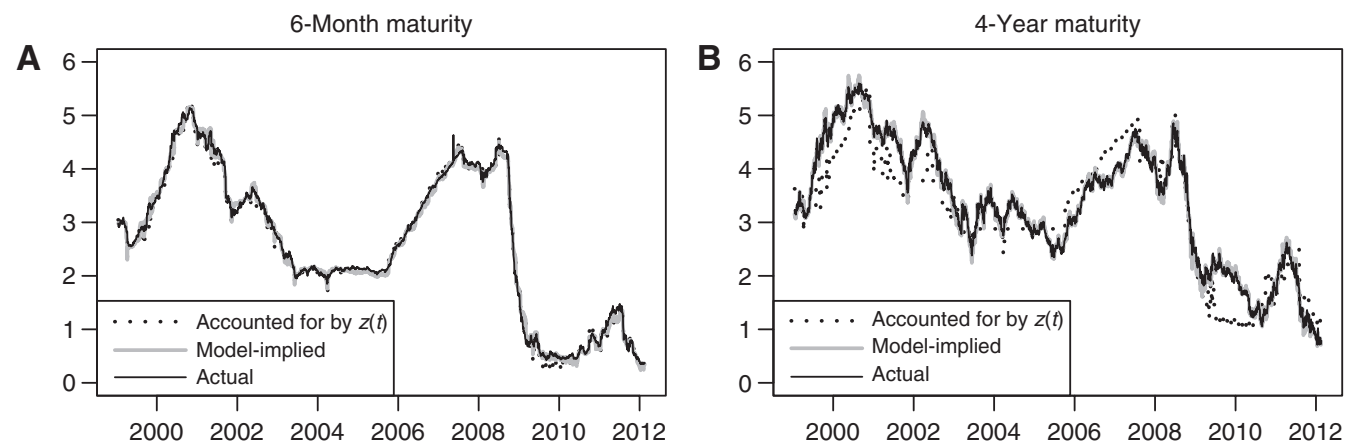

Figure 2: Model fit.

These plots compare model-implied yields with their data (actual) counterparts. The dotted line represents the part of the model-implied yields that is accounted for by the regime variable $z_{t}$ [that is $G(t, h) z_{t}+A_{h}$ in Equation 11].

of the monetary-policy regimes on the term structure of interest rates. Modeled yields are compared with the observed ones for three dates. For each date, three additional yield curves are displayed, each of them corresponding to one of the three monetary-policy regimes. The modeled yield curve corresponds to one of these three curves, the attribution being based on the smoothed probabilities of being in the different 


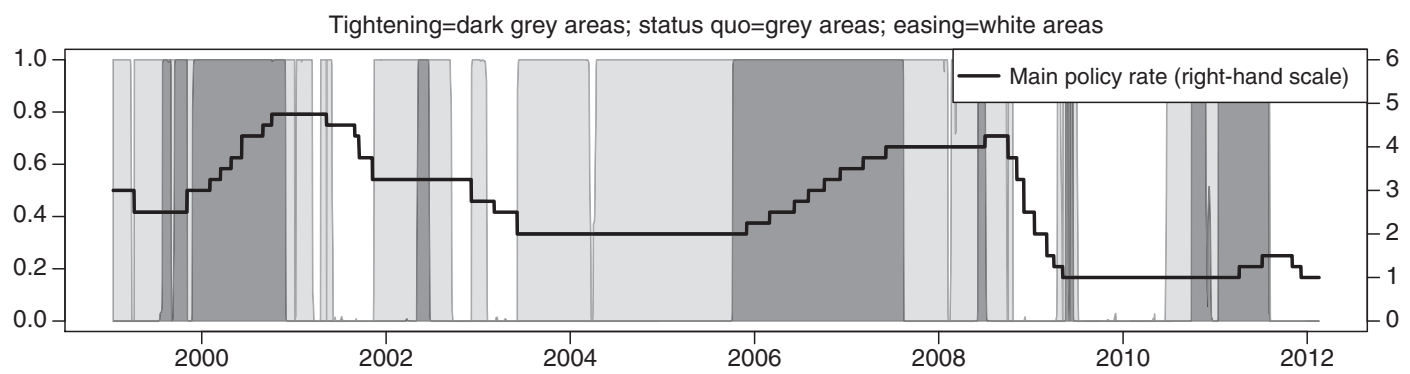

Figure 3: Regimes' estimates.

This figure displays the (smoothed) probabilities of being in the different monetary-policy regimes (left-hand scale). The main policy rate is also shown (right-hand scale).
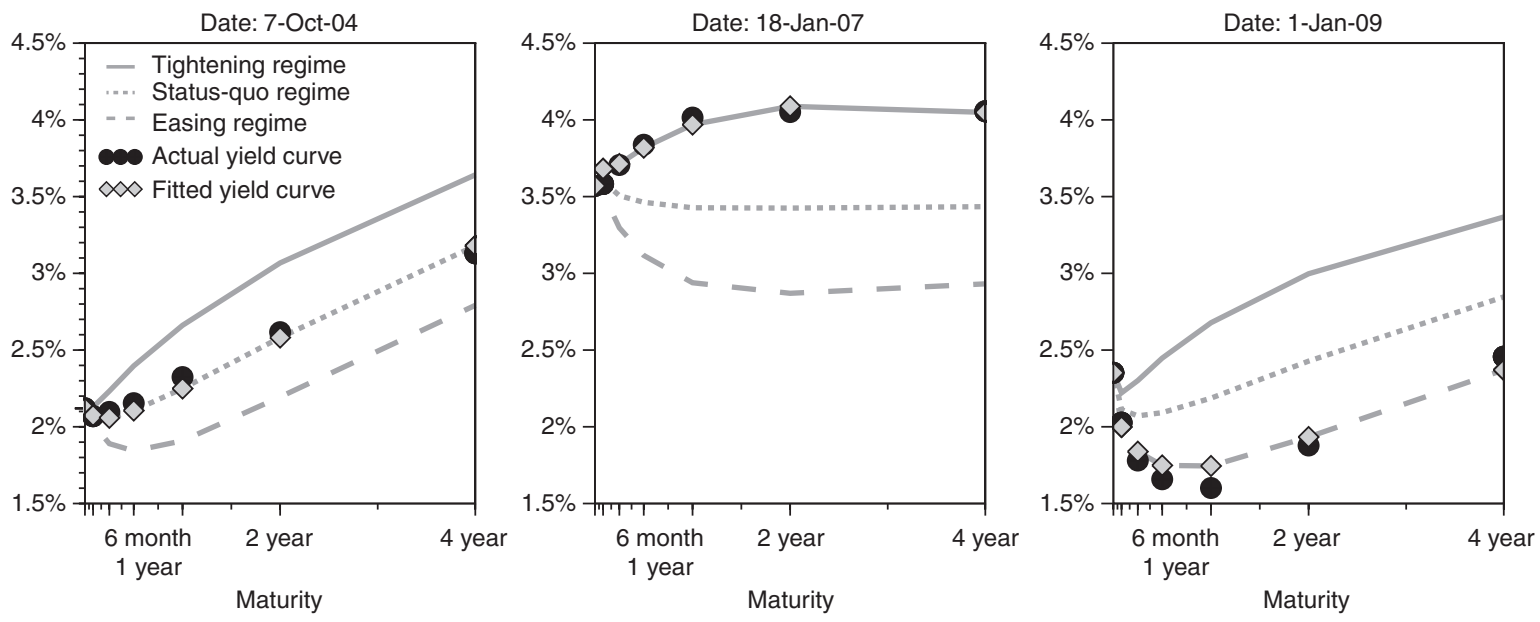

Figure 4: Fitted yield curves and influence of monetary-policy regimes.

These plots compare model-implied (diamonds) with observed (black circles) yield curves at different dates. In addition, each plot reports the (model-implied) yield curves that would have been obtained if other monetary-policy regimes had prevailed on these dates. The seven circles (and diamonds) correspond, respectively to the following maturities: 1 day, 1, 3, 6 months, 1, 2 and 4 years.

monetary-policy regimes. ${ }^{28}$ The two remaining curves are the answers to the question: what if the monetarypolicy stance were different on that date? These plots show that monetary-policy regimes are key to shape the yield curve. Furthermore, this figure demonstrates the ability of the model to reproduce various shapes of the yield curve (steep, flat, humped, inverse-humped).

The specifications of the model allow for an heteroskedastic behavior of the policy rate. This is illustrated in Figure 5, that displays the conditional standard deviations associated to the 3-month-ahead forecasts of the policy rate (i.e. $\left.\sqrt{\operatorname{Var} r_{t}\left[\bar{r}_{t+3 m t h}\right]}\right)$. For any date $t$, these standard deviations depend on (a) the policy rate $\left(\bar{r}_{t}\right)$ and on (b) the monetary-policy regime $\left(z_{m, t}\right)$ prevailing prevailing at date $t$. For instance, the plot suggests that uncertainty characterising future policy rate moves is higher in easing than in tightening phases, which is line with the findings of Fontaine (2009).

28 These probabilities are the ones displayed in Figure 3. In the present case, the smoothing algorithm results in a clear-cut identification of the hidden monetary-policy regime: Most of the time, the smoothed probabilities are either 1 or 0. 


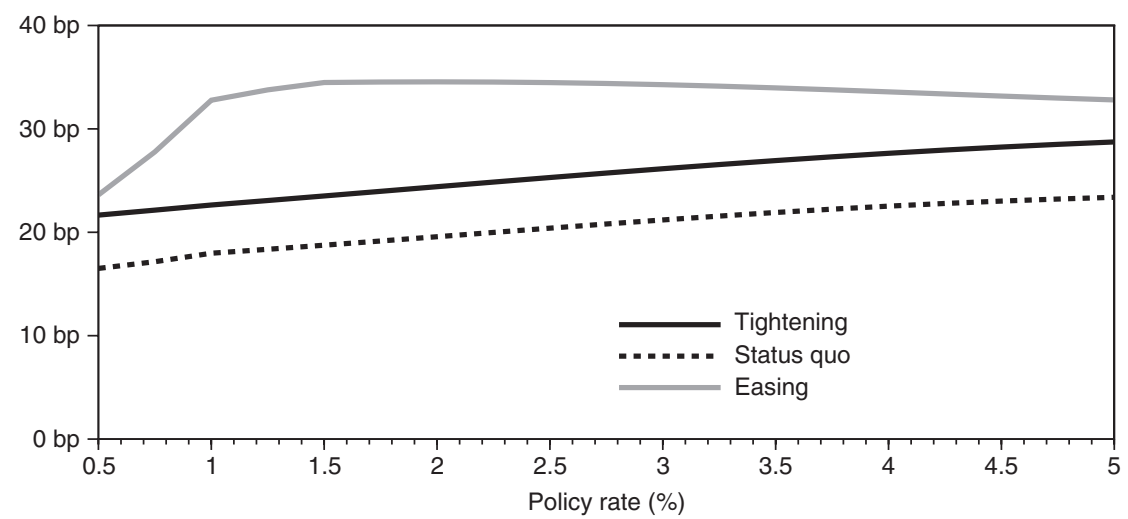

Figure 5: Heteroskedasticity of the policy rate.

This plot presents the conditional standard deviations (reported in basis points) of the 3-month-ahead forecasts of the policy rate - i.e. $\sqrt{\operatorname{Var}_{t}\left(\bar{r}_{t+3 m t h}\right)}$ - as functions of the current policy rate $\left(\bar{r}_{t}\right)$ and of the current monetary-policy regime (easing, status quo or tightening). These standard deviations measure the uncertainty associated to future values of the policy rate. This plot illustrates the heteroskedasticity of the policy rate in the model.

\subsection{Term premiums}

Conceptually, term premiums correspond to average bond excess returns demanded by investors to be compensated for the risk associated with changes in interest rates. Let me clarify. If bond prices tend to be systematically lower - because interest rates tend to be systematically higher - in what investors consider as "bad states of the world", then an investor gets more exposed to these bad states of the world if she purchases the bond. For her to be willing to buy such an asset, the bond price has to be lower than what it would be under the so-called expectation hypothesis, where the preferences of the bond holders would not relate to the level of interest rates (see e.g. Campbell and Shiller (1991) or Cochrane and Piazzesi (2005)). While there is strong evidence against the expectation hypothesis for long-term yields, the evidence is weaker for short-term ones [see Longstaff (2000)].

Formally, in my model, the expectation hypothesis is verified if the prices of risk $v$ and $\delta$ are equal to zero (see the specification of the s.d.f. in Equation 7). In that case, the s.d.f. $M_{t-1, t}$ is equal to $\exp \left(-r_{t-1}\right)$ and the riskneutral and the physical dynamics coincide (because $\left.d \mathbb{Q} / d \mathbb{P}=\exp \left(r_{t-1}\right) M_{t-1, t}\right)$. By contrast, if the preferences of the agents are related to the interest rate risk, the risk-neutral dynamics differs from the physical one and term premiums arise. By definition the term premiums are the differences between model-implied yields $(y(t, h))$ and the counterfactual yields $(\tilde{y}(t, h)$, say) that would be observed if the investors were not averse to interest rate risk. Formally:

$$
\begin{aligned}
& y(t, h)=-\frac{1}{h} \log E_{t}^{\mathbb{Q}}\left(\exp \left[-r_{t}-\ldots-r_{t+h-1}\right]\right) \\
& \tilde{y}(t, h)=-\frac{1}{h} \log E_{t}^{\mathbb{P}}\left(\exp \left[-r_{t}-\ldots-r_{t+h-1}\right]\right) .
\end{aligned}
$$

The existence of term premiums at the short to medium end of the yield curve is important in several respects. Let me mention two of them. First, if these risk premiums are sizeable, OIS forward rates should not be interpreted as the market (physical) expectations of future policy rates for short to medium horizons, though this is the basis of a widespread market practice. ${ }^{29}$ Second, the existence of risk premiums implies that excess returns are partially predictable. ${ }^{30}$

29 See e.g. Barclays (2008), Joyce, Relleen, and Sorensen (2008), Joyce and Meldrum (2009), Bank of England (2005) or Lang (2010).

30 See e.g. Fama and Bliss (1987) and Campbell and Shiller (1991). 

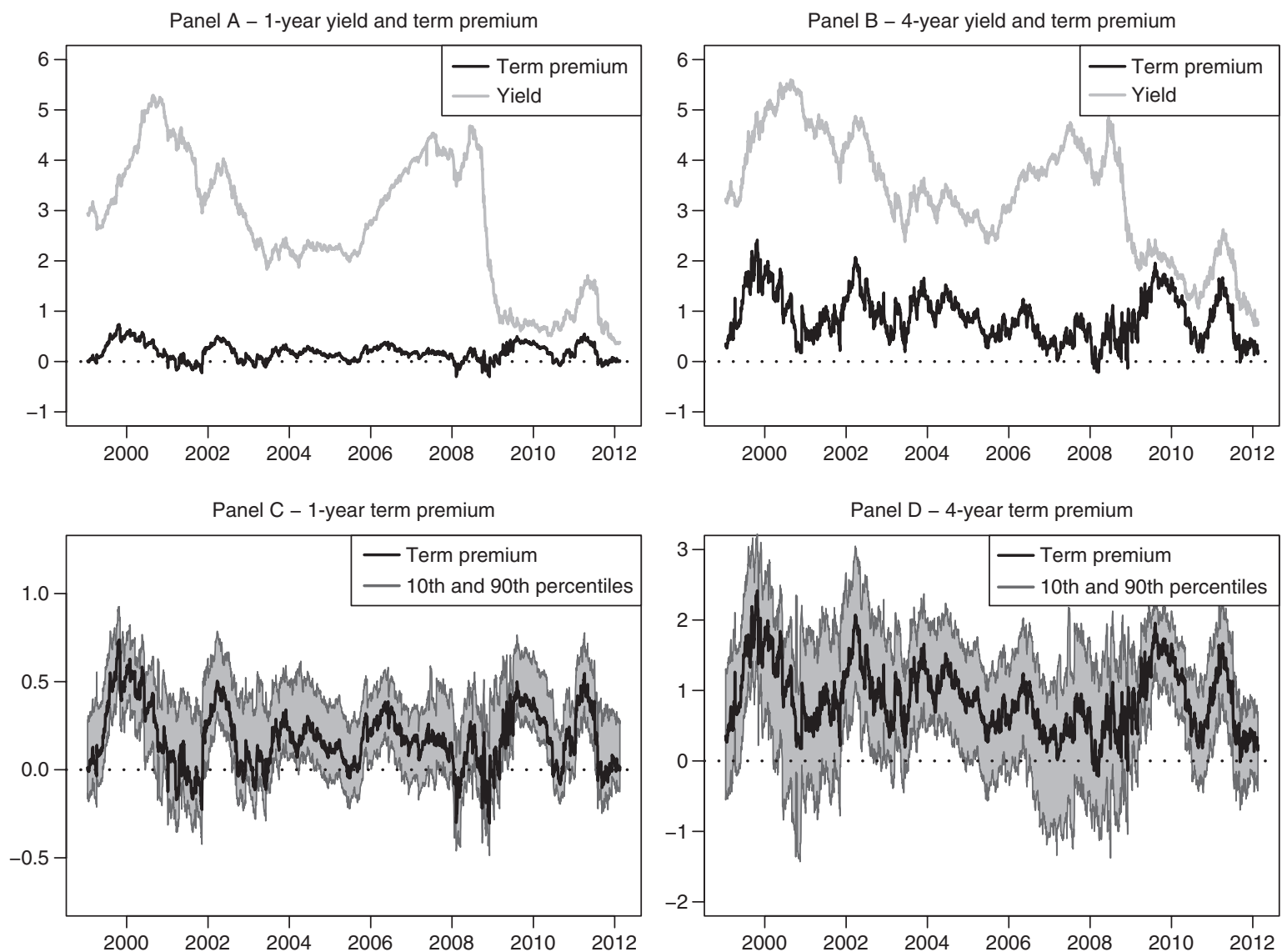

Figure 6: Term premium estimates.

This figure displays the term premium estimates. These term premiums are the parts of the yields that would not exist if agents were not averse to nominal interest rate risks. The upper panels plot the term premiums together with the corresponding yields. The lower plots display $80 \%$ confidence intervals reflecting the uncertainty associated to the parameter estimates. The computation of these confidence intervals is based on bootstrap techniques: the asymptotic distribution of the parameter estimates is used to draw 1000 alternative sets of parameter estimates that, in turn, are used to compute 1000 sets of term premiums; the gray lines indicate the 10 th and 90 th percentiles of the obtained risk-premiums distributions.

Both conditional expectations appearing on the right-hand side of Equations (16) and (17) can be calculated by using the approach detailed in Appendix B. The only difference is that the computation of $y(t, h)$ involves $\Pi_{t}^{*}$ and $\Phi^{*}$, while that of $\tilde{y}(t, h)$ involves $\Pi_{t}$ and $\Phi$ (see Subsection 2.2.1).

Term premiums for two maturities (1 year and 4 years) are plotted on Figure 6. Panels A and B show that the 4-year term premium is higher and more volatile than the 6-month one. This reflects the fact that longer-term instruments are more sensitive than short-term ones to the interest rate risk; investors therefore ask higher premiums to bear that higher risk. At the end of the sample (2009-2012), in a context of very low interest rates, important shares of the yields are accounted for by the term premiums, especially for the 4-year maturity.

Because the term premiums are functions of the model parameterisation, they inherit the uncertainty surrounding parameter estimates. This is illustrated in Panels C and D of Figure 6. These panels display the same term premiums as before, but the 10th and 90th percentiles of their distributions are added. The computation of these percentiles is based on bootstrap techniques. ${ }^{31}$ Naturally, the term premiums are

31 The asymptotic distribution of the parameter estimates is used to draw 1000 alternative sets of parameter estimates that, in turn, are used to compute 1000 sets of term premiums; the gray lines indicate the 10th and 90th percentiles of the obtained riskpremiums distributions (considered date per date). 
statistically significant at the $10 \%$ level when 0 is not included in the confidence interval (gray-shaded area). This happens about half the time, for both the 1-year and the 4-year maturity. These results therefore point to the rejection of the expectation hypothesis, for shorter as for longer maturities.

\section{Conclusion}

While central banks' interest-rate decisions are obvious drivers of the fluctuations of the term structure of interest rates, only few of the available no-arbitrage term structure models feature a realistic modelling of the policy rate. This paper proposes a framework that captures simultaneously the dynamics of the policy rate and that of the yields of longer maturities. Importantly, this model is consistent with the existence of the zero-lower-bound restriction, making it appealing in the current context of extremely low interest rates. A key ingredient of the model is an extensive and innovative use of switching-regime features. Each regime is characterized by a monetary-policy level and a monetary-policy regime (easing, tightening or status quo).

The model is estimated on daily euro-area yield data spanning the period 1999 to 2012 by means of maximum likelihood techniques. The model-implied probabilities of being in the different monetary-policy regimes for any date in the sample constitute interesting by-products of the estimation. Besides, because it involves a stochastic discount factor, the model can be used to compute term premiums. These premiums are the parts of the yields that reflect the excess return demanded by bondholders to bear the interest rate risk. The results suggest that a substantial part of the yields is accounted for by term premiums, which points to the rejection of the expectation hypothesis, even for short-term horizons.

Acknowledgments: I am deeply grateful to Alain Monfort for extremely valuable suggestions and comments. I have benefited from discussions with Simon Dubecq, Jean-Sébastien Fontaine, René Garcia, Rodrigo Guimaraes, Imen Ghattassi, Wolfgang Lemke, Andrew Meldrum, Jean-Stéphane Mésonnier, Emanuel Moench, Benoît Mojon, Fulvio Pegoraro, Marcel Priebsch, Francisco Rivadeynera Sanchez, Michael Rockinger, Thomas Sargent, Andrew Siegel, Min Wei and Paul Whelan. I thank participants at the Fed of San Francisco workshop on term structure modelling at the ZLB (2013), at SoFiE annual meeting (2013), at Canadian Economic Association annual meeting (2012), at AFSE annual meeting (2012), at ESEM annual meeting (2012), at the ECB workshop "Excess liquidity and money-market functioning” (2012), at AFFI Paris finance meeting (2012) and at seminars at Banque de France, Bank of England, HEC Lausanne, EDHEC Business School and Université Paris-Dauphine. I thank Béatrice Saes-Escorbiac and Aurélie Touchais for excellent research assistance. A substantial part of this work was completed when I was at the Banque de France. This paper however expresses my views only; they do not necessarily reflect those of the Banque de France.

\section{Appendix}

\section{A Laplace transform of a Markov-switching process}

In the following, I consider a $n$-state Markov process $z_{t}$, valued in $\left\{e_{1}, \ldots, e_{n}\right\}$, the set of columns of $I_{n}$, the identity matrix of dimension $n \times n$. I assume that the matrix of transition probabilities is deterministic and denoted by $P_{t}$ (the columns sum to one). I have: $\mathbb{P}\left(z_{t+1}=e_{i} \mid z_{t}\right)=e_{i}^{\prime} P_{t+1} z_{t}$. It can be shown that:

$$
\begin{aligned}
E_{t}\left(\exp \left[\alpha_{1}^{\prime} z_{t+1}+\ldots+\alpha_{h}^{\prime} z_{t+h}\right]\right)= & {\left[\begin{array}{lll}
1 & \cdots & 1
\end{array}\right]\left[D\left(\exp \alpha_{h}\right) P_{t+h}\right] \times \ldots } \\
& \ldots \times\left[D\left(\exp \alpha_{1}\right) P_{t+1}\right] z_{t} .
\end{aligned}
$$


where $\exp \alpha$ is the vector whose entries are of the form $\exp \left(\alpha_{i}\right)$ 's and where $D(x)$ is a diagonal matrix whose diagonal entries are the elements of the vector $x$. Indeed, for $h=1$ :

$$
\begin{aligned}
E_{t}\left(\exp \left(\alpha^{\prime} z_{t+1}\right)\right) & =\sum_{i=1}^{n} \exp \left(\alpha_{i}\right) e_{i}^{\prime} P_{t+1} z_{t}=\left(\sum_{i=1}^{M} \exp \left(\alpha_{i}\right) e_{i}^{\prime}\right) P_{t+1} z_{t} \\
& =\left[\begin{array}{lll}
1 & \cdots & 1
\end{array}\right] D(\exp \alpha) P_{t+1} z_{t} .
\end{aligned}
$$

Now, for $h=2$, the law of iterated expectations leads to:

$$
\begin{aligned}
E_{t}\left(\exp \left[\alpha_{1}^{\prime} z_{t+1}+\alpha_{2}^{\prime} z_{t+2}\right]\right) & =E_{t}\left(E_{t}\left[\exp \left[\alpha_{1}^{\prime} z_{t+1}+\alpha_{2}^{\prime} z_{t+2}\right] \mid z_{t+1}\right]\right) \\
& =E_{t}\left(\exp \left[\alpha_{1}^{\prime} z_{t+1}\right] E_{t}\left[\exp \left[\alpha_{2}^{\prime} z_{t+2}\right] \mid z_{t+1}\right]\right)
\end{aligned}
$$

Then, using the previous case:

$$
\begin{aligned}
E_{t}\left(\exp \left[\alpha_{1}^{\prime} z_{t+1}+\alpha_{2}^{\prime} z_{t+2}\right]\right) & =E_{t}\left(\exp \left[\alpha_{1}^{\prime} z_{t+1}\right][1 \cdots 1] D\left(\exp \alpha_{2}\right) P_{t+2} z_{t+1}\right) \\
& =E_{t}\left(\left[\begin{array}{lll}
1 & \cdots & 1
\end{array}\right] D\left(\exp \alpha_{2}\right) P_{t+2} z_{t+1} \exp \left[\alpha_{1}^{\prime} z_{t+1}\right]\right) \\
& =E_{t}\left(\left[\begin{array}{llll}
1 & \cdots & 1
\end{array}\right] D\left(\exp \alpha_{2}\right) P_{t+2} z_{t+1} z_{t+1}^{\prime} D\left(\exp \alpha_{1}\right)\left[\begin{array}{lll}
1 & \cdots & 1
\end{array}\right]^{\prime}\right)
\end{aligned}
$$

Using the facts that $z_{t+1} z_{t+1}^{\prime}$ commutes with any matrix and that $z_{t+1} z_{t+1}^{\prime}[1 \cdots 1]^{\prime}=z_{t+1}$, I get:

$$
\begin{aligned}
E_{t}\left(\exp \left[\alpha_{1}^{\prime} z_{t+1}+\alpha_{2}^{\prime} z_{t+2}\right]\right) & =E_{t}\left(\left[\begin{array}{lll}
1 & \cdots & 1
\end{array}\right] D\left(\exp \alpha_{2}\right) P_{t+2} D\left(\exp \alpha_{1}\right) z_{t+1}\right) \\
& =\left[\begin{array}{lll}
1 & \cdots & 1
\end{array}\right]\left[D\left(\exp \alpha_{2}\right) P_{t+2}\right]\left[D\left(\exp \alpha_{1}\right) P_{t+1}\right] z_{t} .
\end{aligned}
$$

\section{B Pricing formulas}

In this Appendix, I briefly explain how to compute the three terms $P_{z}(t, h), P_{\xi}(t, h)$ and $P_{s}(t, h)$ whose product is the bond price $P(t, h)$ [Equation (10)].

\section{B.1 Computation of $P_{z}(t, h)$}

The targets $\bar{r}_{t}$ are the only stochastic variables involved in the computation of $P_{1},(t, h)$. The previous Appendix shows that the expectation of an exponential-affine combination of a variable that follows a Markov-switching process is available in closed form. This leads to the following formula:

$$
\begin{gathered}
P_{z}(t, h)=E_{t}^{\mathbb{Q}}\left(\exp \left[-\sum_{i=0}^{h-1} \bar{r}_{t+i}\right]\right)=G_{z}(t, h) z_{t} \\
\text { with } G_{z}(t, h)=\left[\begin{array}{lll}
1 & \cdots & 1
\end{array}\right]\left[\prod_{i=h-1}^{1} D\left(\exp \left[-\Delta_{m}\right]\right) \Pi_{t+i}^{*}\right] D\left(\exp \left[-\Delta_{m}\right]\right)
\end{gathered}
$$

and where the product operator $\Pi$ works in a backward direction: if $X_{1}$ and $X_{2}$ are some square matrices, $\prod_{i=2}^{1} X_{i}=X_{2} X_{1}$. Defining the vector $G(t, h)$ as $G(t, h)=-(1 / h) \log \left\{G_{z}(t, h)\right\}$, I get $P_{z}(t, h)=\exp \left(-h G(t, h) z_{t}\right)$.

\section{B.2 Computation of $P_{\xi}(t, h)$}

Using the independence assumption of the $\xi_{t} \mathrm{~s}$, I obtain:

$$
E_{t}^{\mathbb{Q}}\left(\exp \left[-\sum_{i=0}^{h-1} \xi_{t+i}\right]\right)=\exp \left(-\xi_{t}\right)\left[E_{t}^{\mathbb{Q}}\left(\exp \left[-\xi_{t+1}\right]\right)\right]^{h-1} .
$$


According to the definition of the $\mathscr{L}$ distribution (see Subsection 2.1.2), the expectation $E_{t}^{\mathbb{Q}}\left(\exp \left[-\xi_{t+1}\right]\right)$ is given by:

$$
\exp (-w)\left\{p \times \mathscr{F}\left(\alpha_{P}, \beta_{P},-1\right)+(1-p) \times \mathscr{F}\left(\alpha_{N}, \beta_{N}, 1\right)\right\}
$$

where $\mathscr{F}(\alpha, \beta, v)$ is given by: $\mathscr{F}(\alpha, \beta, v)=1+\sum_{k=0}^{\infty} \frac{v^{k}}{k !}\left(\prod_{i=0}^{k-1} \frac{\alpha+i}{\alpha+\beta+i}\right)$ (using the Laplace transform of the Beta distribution). Note that $\exp \left(-\xi_{t}\right) / E_{t}^{\mathbb{Q}}\left(\exp \left[-\xi_{t+1}\right]\right)$ is very close to one (especially at the daily frequency). With virtually no impact on the result, I therefore use the approximation:

$$
P_{\xi}(t, h)=E_{t}^{\mathbb{Q}}\left(\exp \left[-\sum_{i=0}^{h-1} \xi_{t+i}\right]\right) \approx\left[E_{t}^{\mathbb{Q}}\left(\exp \left[-\xi_{t+1}\right]\right)\right]^{h}=: \exp \left(-h A_{h}^{(\xi)}\right)(\text { say }) .
$$

\section{B.3 Computation of $P_{s}(t, h)$}

We have

$$
\begin{gathered}
P_{s}(t, h)=E_{t}^{\mathbb{Q}} e^{-\sum_{i=0}^{h-1} s_{t+i}}=E_{t}^{\mathbb{Q}} e^{-\sum_{i=0}^{h-1} s_{1, t+i}+s_{2, t+i}} \\
\text { where }\left[\begin{array}{l}
s_{1, t} \\
s_{2, t}
\end{array}\right]=\Phi^{*}\left[\begin{array}{l}
s_{1, t-1} \\
s_{2, t-1}
\end{array}\right]+\Sigma \varepsilon_{t}^{*}, \varepsilon_{t}^{*} \sim \text { i.i.d. } \mathscr{N}^{\mathbb{Q}}(0, I) .
\end{gathered}
$$

The previous equation can be solved using the recursive algorithm proposed by Ang and Piazzesi (2003). A faster computation can be obtained by using the algorithm described in Borgy et al. (2011). The resulting price is of the form:

$$
P_{s}(t, h)=\exp \left(-h A_{h}^{(s)}-h B_{h} s_{t}\right)
$$

\section{Computation of the likelihood}

Multiplying both sides of Equation (13) by $\left(\Lambda_{s}^{\prime} \Lambda_{s}\right)^{-1} \Lambda_{s}^{\prime}$, I obtain:

$$
\left(\Lambda_{s}^{\prime} \Lambda_{s}\right)^{-1} \Lambda_{s}^{\prime} R_{t}=\left(\Lambda_{s}^{\prime} \Lambda_{s}\right)^{-1} \Lambda_{s}^{\prime} \Lambda_{z} z_{t}+s_{t}+\left(\Lambda_{s}^{\prime} \Lambda_{s}\right)^{-1} \Lambda_{s}^{\prime} \eta_{t}
$$

The assumption according to which $s_{t}=\left(\Lambda_{s}^{\prime} \Lambda_{s}\right)^{-1} \Lambda_{s}^{\prime}\left(R_{t}-\Lambda_{z} z_{t}\right)$, which reflects the fact the linear combination of yields $\left(\Lambda_{s}^{\prime} \Lambda_{s}\right)^{-1} \Lambda_{s}^{\prime} R_{t}$ is priced without error, implies that the last term in the previous equation is null, that is: $\Lambda_{s}^{\prime} \eta_{t}=0$. This notably implies that the elements of $\eta_{t}$ can then not be independent. However, the elements of a subset of $M-1$ of them can be independent. Without loss of generality, I assume that the last $M-1$ elements of $\eta_{t}$ are i.i.d. $\mathscr{N}\left(0, \sigma_{R}^{2}\right)$.

For a given regime vector $z_{t}$, there is the same information in $R_{t}$ as in $\left\{s_{t}, \tilde{R}_{t}\right\}$, where $\tilde{R}_{t}$ is any subvector of $R_{t}$ containing $M-1$ yields of distinct maturities. My intermediary objective is to compute the likelihood associated to $\left\{s_{t}, \tilde{R}_{t}\right\}_{t=1, \ldots, T}$. Without loss of generality, I assume that $\tilde{R}_{t}=\left[y\left(t, h_{2}\right), \ldots, y\left(t, h_{M}\right)\right]^{\prime}$. Denoting by $\Gamma$ the $(M-1) \times M$ matrix that selects the last $M-1$ entries of an $M \times 1$ vector, I have $\tilde{R}_{t}=\Gamma R_{t}$. Substituting for $s_{t}$ in Equation (14) and rearranging the terms in this equation leads to:

$$
\eta_{t}=(I d-\Lambda) R_{t}-(I d-\Lambda) \Lambda_{m, t} z_{m, t},
$$

where $\Lambda=\Lambda_{s}\left(\Lambda_{s}^{\prime} \Lambda_{s}\right)^{-1} \Lambda_{s}^{\prime}$. Multiplying both sides of the previous equation by $\Gamma$ gives:

$$
\tilde{\eta}_{t}=\Gamma(I d-\Lambda) R_{t}-\Gamma(I d-\Lambda) \Lambda_{m, t} z_{m, t},
$$

where $\tilde{\eta}_{t} \equiv \Gamma \eta_{t}$ is the vector containing the last $M-1$ (i.i.d.) elements of $\eta_{t^{*}}$ 
Besides, let me rewrite the second equation of System (6) after substituting for $s_{t}\left(\equiv S_{2, t}\right)$ and $s_{t-1}\left(\equiv S_{2, t-1}\right)$ :

$$
\varepsilon_{2, t}=\frac{1}{\sigma_{s}}\left(\Lambda_{s}^{\prime} \Lambda_{s}\right)^{-1} \Lambda_{s}^{\prime}\left[\left(R_{t}-\rho_{2} R_{t-1}\right)-\Lambda_{z}\left(z_{t}-\rho_{2} z_{t-1}\right)\right],
$$

where $\varepsilon_{2, t} \sim \mathcal{N}(0,1)$ (this is the second element of $\left.\varepsilon_{t}\right)$. The system made of Equations (21) and (22) involves a set of independent Gaussian shocks $\left\{\varepsilon_{2, t}, \eta_{2, t}, \ldots, \eta_{M, t}\right\}$ as well as the (unobserved) regime variable $z_{m, t}$. The computation of the log-likelihood associated with the previous system of equations is obtained by applying the Kitagawa-Hamilton filter (see e.g. Hamilton (1994), Chapter 22). However, this likelihood is the one associated with the vector $\left\{s_{t}, \tilde{R}_{t}\right\}_{t=1, T}$, while I need to maximize the one associated with actually observed data $\left\{R_{t}\right\}_{t=1, \ldots, T}$. The latter likelihood is obtained by multiplying the former by the determinant of the Jocobian resulting from this change in variables, that is $\left|\partial\left[s_{t}, \tilde{R}_{t}^{\prime}\right] / \partial R_{t}\right|=\frac{1}{\Lambda_{s}^{\prime} \Lambda_{s}} \Lambda_{s, 1}$ where $\Lambda_{s, 1}$ is the first entry of $\Lambda_{s}$.

\section{References}

Andreasen, Martin, and Andrew Meldrum. "Likelihood Inference in Non-Linear Term Structure Models: The Importance of the Zero Lower Bound.” BoE Working Papers, Bank of England 2011.

Ang, Andrew, and Monika Piazzesi. 2003. "A No-Arbitrage Vector Autoregression of Term Structure Dynamics with Macroeconomic and Latent Variables." Journal of Monetary Economics 50 (4): 745-787.

Ang, Andrew, Geert Bekaert, and Min Wei. 2008. "The Term Structure of Real Rates and Expected Inflation.” Journal of Finance 63 (2): 797-849.

Bank of England. 2005. “Markets and Operations.” In Bank of England Quarterly Bulletin, Winter, Bank of England, 407-423. Bansal, Ravi, and Hao Zhou. 2002. “Term Structure of Interest Rates with Regime Shifts.” Journal of Finance 57 (5): $1997-2043$. Barclays. 2008. EONIA Swaps: Definition, Uses and Advantages. European Fixed Income Strategy, Barclays.

Beirne, John. 2012. "The Eonia Spread Before and During the Crisis of 2007-2009: the Role of Liquidity and Credit Risk." Journal of International Money and Finance 31 (3): 534-551.

Bikbov, Ruslan, and Mikhail Chernov. 2008. “Monetary Policy Regimes and the Term Structure of Interest Rates.” CEPR Discussion Papers, C.E.P.R. Discussion Papers.

BIS. 2013. “Towards Better Reference Rate Practices: a Central Bank Perspective.” BIS Report, Bank for International Settlements.

Black, Fischer. 1995. “Interest Rates as Options.” Journal of Finance 50 (5): 1371-1376.

Borgy, Vladimir, Thomas Laubach, Jean-Stephane Mesonnier, and Jean-Paul Renne. 2011. "Fiscal Sustainability, Default Risk and Euro Area Sovereign Bond Spreads Markets." Working paper series 350, Banque de France.

Campbell, John Y., and Robert J. Shiller. 1991. "Yield Spreads and Interest Rate Movements: A Bird's Eye View." Review of Economic Studies 58 (3): 495-514.

Chen, Ren-Raw, and Louis Scott. 1993. "Maximum Likelihood Estimation for a Multifactor Equilibrium Model of the Term Structure of Interest Rates." Journal of Fixed Income 3: 14-31.

Christensen, Jens H. E., and Glenn D. Rudebusch. 2013. "Modeling yields at the zero lower bound: are shadow rates the solution?.” Working Paper Series 2013-39, Federal Reserve Bank of San Francisco.

Christensen, Jens H.E., and Glenn D. Rudebusch. 2015. “Estimating Shadow-Rate Term Structure Models with Near-Zero Yields.” Journal of Financial Econometrics 13 (2): 226-259.

Cochrane, John H., and Monika Piazzesi. 2002. "The Fed and Interest Rates - A High-Frequency Identification." American Economic Review 92 (2): 90-95.

Cochrane, John H., and Monika Piazzesi. 2005. “Bond Risk Premia.” American Economic Review 95 (1): 138-160.

Cox, John C., Jonathan E. Ingersoll, and Stephen A. Ross. 1985. “An Intertemporal General Equilibrium Model of Asset Prices.” Econometrica 53 (2): 363-384.

Dai, Qiang, and Kenneth J. Singleton. 2003. “Term Structure Dynamics in Theory and Reality.” Review of Financial Studies 16 (3): 631-678.

Dai, Qiang, Kenneth J. Singleton, and Wei Yang. 2007. "Regime Shifts in a Dynamic Term Structure Model of U.S. Treasury Bond Yields.” Review of Financial Studies 20 (5): 1669-1706.

Dubecq, Simon, and Christian Gouriéroux. 2011. "An Analysis of the Ultra Long-Term Yields.” mimeo.

Fahr, Stephan, Roberto Motto, Massimo Rostagno, Frank Smets, and Oreste Tristani. 2010. "Lessons for Monetary Policy Strategies from the Recent Past.” paper presented at the 6th EBC Central Banking Conference, European Central Bank.

Fama, Eugene F., and Robert R. Bliss. 1987. “The Information in Long-Maturity Forward Rates.” American Economic Review 77 (4): 680-692. 
Fontaine, Jean-Sebastien. 2009. “Fed Funds Futures and the Federal Reserve.” Working Papers, Bank of Canada.

Garcia, René, and Pierre Perron. 1996. "An Analysis of the Real Interest Rate under Regime Shifts.” The Review of Economics and Statistics 78 (1): 111-125.

Gray, Stephen F. 1996. "Modeling the Conditional Distribution of Interest Rates as a Regime-switching Process." Journal of Financial Economics 42 (1): 27-62.

Hamilton, James D. 1988. "Rational-Expectations Econometric Analysis of Changes in Regime: An Investigation of the Term Structure of Interest Rates." Journal of Economic Dynamics and Control 12 (2-3): 385-423.

Hamilton, James D. 1994. Time Series Analysis, princeton university press ed.

Hansen, Lars Peter and Scott F. Richard. 1987. “The Role of Conditioning Information in Deducing Testable.” Econometrica 55 (3): 587-613.

Joyce, Michael, and Andrew Meldrum. 2009. “Market Expectations of Future Bank Rate.” In Quarterly Bulletin 2008 Q3. Bank of England, pp. 274-282.

Joyce, Michael, Jonathan Relleen, and Steffen Sorensen. 2008. "Measuring Monetary Policy Expectations from Financial Market Instruments." Bank of England working papers 356, Bank of England.

Joyce, Michael, Ana Lasaosa, Ibrahim Stevens, and Matthew Tong. 2011. "The Financial Market Impact of Quantitative Easing in the United Kingdom." International Journal of Central Banking 7 (3): 113-161.

Kahn, George A. 2010. “Monetary Policy under a Corridor Operating Framework.” Economic Review (Q4) 95 (4): 5-34.

Kim, Chang-Jin. 1994. “Dynamic Linear Models with Markov-Switching.” Journal of Econometrics 60 (1-2): 1-22.

Kim, Don H., and Kenneth J. Singleton. 2012."Term Structure Models and the Zero Bound: An Empirical Investigation of Japanese Yields." Journal of Econometrics 170 (1): 32-49.

Kim, Don H., and Marcel A. Priebsch. 2013. "Estimation of Multi-Factor Shadow-Rate Term Structure Models.” Technical Report, Board of Governors of the Federal Reserve System (U.S.).

Krippner, Leo. 2013. “Measuring the Stance of Monetary Policy in Zero Lower Bound Environments.” Economics Letters 118 (1): 135-138.

Lang, Ian. 2010. “Interest Rate Derivatives.” In Financial Derivatives, edited by Robert W. Kolb and James A. Overdahl, John Wiley \& Sons, chapter 10, 389-472.

Lemke, Wolfgang, and Theofanis Archontakis. 2008. "Bond Pricing when the Short-Term Interest Rate Follows a Threshold Process." Quantitative Finance 8 (8): 811-822.

Longstaff, Francis A. 2000. "The Term Structure of Very Short-term Rates: New Evidence for the Expectations Hypothesis." Journal of Financial Economics 58 (3): 397-415.

Monfort, Alain, and Jean-Paul Renne. 2013. “Default, Liquidity and Crises: An Econometric Framework.” Journal of Financial Econometrics 11 (2): 221-262.

Piazzesi, Monika. 2005. “Bond Yields and the Federal Reserve.” Journal of Political Economy 113 (2): 311-344.

Piazzesi, Monika. 2010. “Affine Term Structure Models." In Handbook of Financial Econometrics, Volume 1, edited by Yacine Ait-Sahalia and Lars Peter Hansen, North Holland: Elsevier, chapter 12, 389-472.

Priebsch, Marcel A. 2013. "Computing Arbitrage-Free Yields in Multi-Factor Gaussian Shadow-Rate Term Structure Models." Finance and Economics Discussion Series 2013-63, Board of Governors of the Federal Reserve System (U.S.).

Renne, Jean-Paul. 2016. “A Tractable Interest Rate Model wif Explicit Monetary Policy Rates.” European Journal of Operational Research 251: 873-887.

Smaghi, Bini. 2009. “Three Question on Monetary-Policy Easing.” Lecture at the University of Ancona, 6 March 2009, European Central Bank.

Soares, Carla, and Paulo M. M. Rodrigues. 2013. "Determinants of the Eonia Spread and the Financial Crisis.” The Manchester School 81 (S3): 82-110.

Wu, Jing Cynthia, and Fan Dora Xia. 2013. "Measuring the Macroeconomic Impact of Monetary Policy at the Zero Lower Bound.” Working Papers, Chicago Booth.

Supplemental Material: The online version of this article (DOI: 10.1515/snde-2016-0043) offers supplementary material, available to authorized users. 